\title{
A Novel 3D Partitioned Active Shape Model for Segmentation of Brain MR Images
}

\author{
Zheen Zhao ${ }^{1,2}$, Stephen R. Aylward ${ }^{1}$, and Eam Khwang Teoh ${ }^{2}$ \\ 1 Computer Aided Display and Diagnosis Laboratory, \\ University of North Carolina at Chapel Hill, Chapel Hill, NC \\ kurtzhao@unc.edu, aylward@unc.edu http://caddlab.rad.unc.edu \\ 2 School of Electrical \& Electronics Engineering, \\ Nanyang Technological University, Singapore \\ ekteoh@ntu.edu.sg
}

\begin{abstract}
A 3D Partitioned Active Shape Model (PASM) is proposed in this paper to address the problems of the 3D Active Shape Models (ASM). When training sets are small. It is usually the case in 3D segmentation, 3D ASMs tend to be restrictive. This is because the allowable region spanned by relatively few eigenvectors cannot capture the full range of shape variability. The 3D PASM overcomes this limitation by using a partitioned representation of the ASM. Given a Point Distribution Model (PDM), the mean mesh is partitioned into a group of small tiles. In order to constrain deformation of tiles, the statistical priors of tiles are estimated by applying Principal Component Analysis to each tile. To avoid the inconsistency of shapes between tiles, training samples are projected as curves in one hyperspace instead of point clouds in several hyperspaces. The deformed points are then fitted into the allowable region of the model by using a curve alignment scheme. The experiments on 3D human brain MRIs show that when the numbers of the training samples are limited, the 3D PASMs significantly improve the segmentation results as compared to 3D ASMs and 3D Hierarchical ASMs.
\end{abstract}

\section{Introduction}

Object segmentation is a prerequisite of many medical image analysis applications. The 2D Active Shape Model (ASM) [1] provide a promise to improve the robustness of the model to local minimum by restricting the deformation within the allowable region.

However, in 3D segmentation, 3D ASM often constrains itself from catching details during deformations. This is because the number of eigenvectors/eigenmodes cannot exceed the number of training samples, in the case of the dimension of the model being large, which is usually the case in 3D segmentation, as the dimension of the model is typically two or three orders of magnitude higher than the number of training samples. It is difficult to estimate a high-dimensional probability distribution from a small training set. Therefore, the allowable region spanned by the relatively few eigenvectors limits the deformation of ASM to catch details. A solution is by using large training sets. However, it requires 
manually segmenting 3D images slice by slice, which is very laborious. Therefore it is inconvenient to build a large training set.

There have been various attempts to address this limitation. ASM combined with elastic models has been proposed in [1], but the detailed deformations are regulated by elastic forces, which do not reflect true shape variability. In [2, Davatzikos et al. proposed 2D Hierarchical Active Shape Model (HASM), a hierarchical scheme based on 2D curve partition and 2D curve wavelet decomposition to keep details. Their experiments have shown promising results in this "short of samples" scenario.

However, in [2], the spatially partitioned representations of objects, both curve segmentation and wavelet decomposition, introduce shape inconsistency. Because in [2], the allowable regions/plausible areas of bands are independent to each other, illegal shapes could be tolerated during model fitting. As a result, this model could be vulnerable to noises and low contrasts. The authors use a hierarchical representation and a deformation scheme to subdue this problem. However the approaches only partially solve this problem.

Another issue of HASM in [2] is that the distribution is assumed as Gaussian distribution. In 3D applications, this assumption is debatable because the number of training samples is usually so limited that the distribution is sparse. For non-Gaussian distributions, Cootes uses a mixture of Gaussians approximation to a kernel density estimate of the distribution in 11. But for partitioned representations, sole non-linear estimation is not adequate, because the estimations are still independent between tiles/bands.

In this work, a 3D Partitioned Active Shape Model, which uses curve alignment to fit models during deformations, is proposed. In 3D PASM, each training sample and deformed model is represented as a curve instead of a point as ASM and HASM. After the model has deformed to find the best match in the image, the deformed model is projected back. The curve representing the deformed model is aligned with the closest training sample by undergoing an affine transformation. Furthermore, to investigate the strength and weakness of 3D PASM, the 2D HASM in [2] is extended to 3D HASM in this work based on the mesh partitioning algorithm proposed in this paper.

\section{A 3D Partitioned Active Shape Model}

A 3D Partitioned Active Shape Model is described in this section. It is assumed the vertices of manual segmentations have been corresponded to construct a Point Distribution Model (PDM). It means that $N$ training samples are available as sets of $K$ corresponding landmarks in the 3D space.

\subsection{Construction of Allowable Regions}

In conventional ASMs, the training samples are aligned by using Procrustes alignment. The vectors $X_{n}, n=1, \ldots, N$ are then formed by concatenating the coordinates of $K$ landmark points of $N$ samples. Therefore, the dimension of $X$ 
is $3 K \times N$. To reduce the dimension of $X$, Principal Component Analysis (PCA) is applied to $X$. The eigenvectors $e_{0}, \ldots, e_{N-1}$, which are corresponding to the nonzero eigenvalues of the covariance matrix of $X$, are calculated. In the $3 \mathrm{D}$ case, it is typical that $N \ll 3 K$. Therefore it is likely that $S$, spanned by $e_{0}, \ldots, e_{N-1}$, cannot include the full range of shape variation. As a result, 3D ASM, which projects the tentative shape by using $S$, tends to reconstruct a shape without fine details.

3D PASM solves this problem by a partitioned representation of 3D ASMs. Because a 3D ASM is represented as a mean mesh and its eigen variations of individual tiles, the partitioned representation of the ASM is the partitioned mean mesh and eigen variations of tiles. Mesh partition means that a mesh is partitioned into a group of surface patches, which are called tiles in this study.

If only the statistical prior of a small tile, which comprises of $K^{\prime}$ vertices, of the whole mesh is required, $N$ samples will be adequate to include the variation of this tile, as long as the $K^{\prime}$ is small enough. Thus fine details of this tile could be captured in the allowable region.

Therefore, the mesh is partitioned into tiles so that the tiles cover all faces of the mesh and each tile consists of roughly $K^{\prime}$ vertices. PCA is then independently applied to each tile to form a statistical prior for each tile.

\subsection{Mesh Partitioning}

In this section, an algorithm is described to partition a mesh $M$ into a group of tiles. A result of applying this algorithm is shown in Fig. 1, where the tiles are shown in red and the boundaries are shown in blue. This algorithm segments mesh $M$ into tiles $\tau_{1}, \ldots, \tau_{s}$ which cover all faces of $M$, given a set of sites positioned at the centroids of the site faces $S=f_{1}, \ldots, f_{s}$. A face of $M$ is randomly selected as the initial site. Once the tile associated with the site stops growing, another face of $M$ from the faces not covered by grown tiles is selected as the next site. The procedure is repeated until all faces of $M$ are covered by tiles. In other words, the algorithm stops when all faces of $M$ are included in tiles.

A tile $\tau_{i}$ is a collection of faces for which the closest site face is $f_{i}$. The measure of distance between faces is an approximation of the geodesic distance over the mesh. It is defined by constructing a dual directed graph of the mesh $M$, i.e. the nodes of the graph correspond to faces of $M$. The edges of the graph

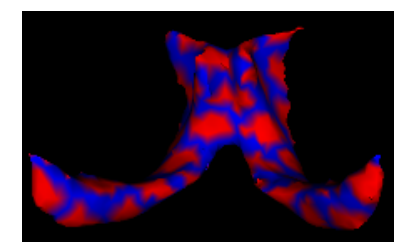

Fig. 1. A partitioned mesh. Tiles are shown in red; boundaries are shown in blue. The blue boundaries are enlarged for visualization. 
connect nodes of adjacent faces. The cost of edges in this directed graph is set to the distance between centroids of the corresponding faces. This distance is defined as the length of the path in this directed graph.

Constructing a tile is a single-source shortest path problem in the graph, which is solved by a variant of Dijkstra's algorithm . The algorithm grows a tile until the size of the tile reaches the maximum. The size of a tile is defined as the number of vertices included in the faces of the tile. The maximum is set by $K_{\max }^{\prime}=\sqrt{K} \times 1.2$, where the $K$ is the number of vertices of mesh $M$. The $K_{\max }^{\prime}$ is set so that the number of tiles is not much bigger than the sizes of individual tiles. More partitioned objects are available at www.unc.edu/ kurtzhao.

\subsection{Model Fitting Scheme}

In ASM, after applying PCA, each training sample is represented as one point in a hyperspace. The training set constructs a cloud in this space. The allowable region can be estimated from the distribution of this cloud of points/samples.

In PASMs, PCA is independently applied to the coordinates of vertices of each tile. Each training sample is then represented as points in $H$ hyperspaces, one point in each hyperspace. In order to represent samples in one hyperspace, the indices of tiles are introduced as another dimension, the $H$ hyperspaces are then combined to one hyperspace. As a result, each training sample is represented as a curve in the new hyperspace instead of points in many hyperspaces. The curves are called sample curves. During deformation, the model points move to find the best match in a test image. The deformed model is then projected back to this hyperspace as a curve called the model curve. The closest sample curve, in terms of Euclidean distance, is chosen as the target. The model curve is then aligned with the target by undergoing an affine transformation. The affine invariant alignment can be estimated by using the Least Square method in [3. The affine transformation is chosen because it strike a balance between keeping the shape of the model plausible and allowing the model approaching the target.

The model fitting scheme of the 3D PASM is shown in the left figure in Fig. 2 where solid lines indicate models, the broken lines stand for training samples. Assume that the model/mesh is partitioned into 3 tiles and the dimension of the 3 hyperspaces is 1D after individual PCA applications. The indices of the tiles are introduced as another dimension. The two training samples are then represented by two curves. To fit the deformed model points, the curve representing the deformed model is aligned with the curve representing training sample 1 by using an affine transformation, which is the closest sample to the model curve. As a result, the shape of the fitted model cannot be far from the closest sample. The inconsistency between tiles is avoided, because the relationship between the shapes of tiles from the samples are enforced. On the contrary, the model fitting of the traditional HASM 2] and the 3D HASM shown in the right figure in Fig. 2 treats training samples as individual points in individual hyperspaces of the tiles. The deformed model points are fitted to the model by a truncation. It could tolerate the inconsistency of shapes because the shape of the fitted model could be different from any training sample. 


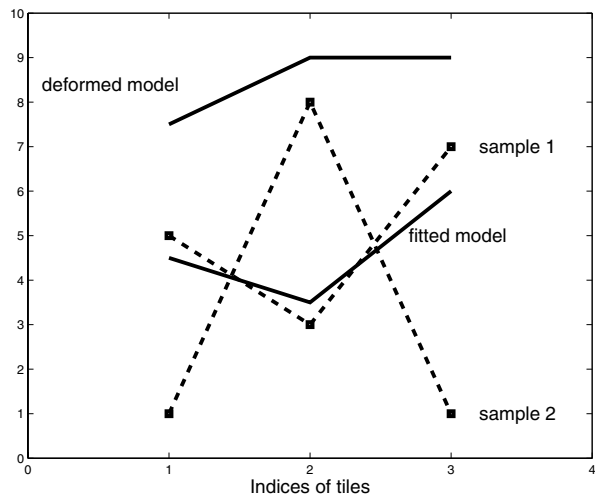

3D PASM

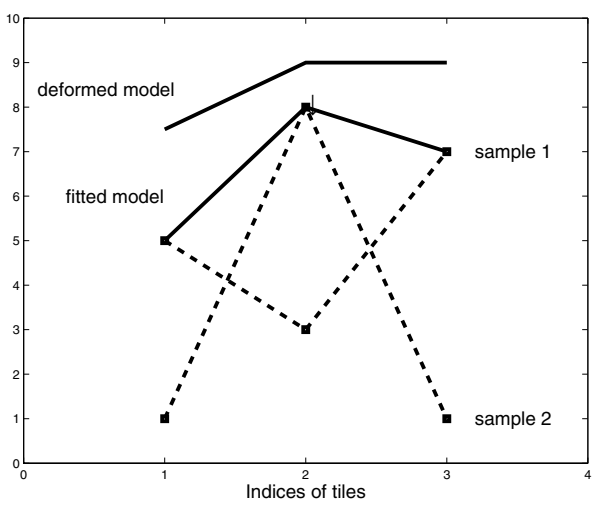

3D HASM

Fig. 2. The model fitting schemes of the 3D PASM and the 3D HASM

There are two forms of 2D HASM. One uses curve segmenting and the other one use wavelet decomposition. The 3D extension of the 2D HASM uses the mesh partitioning instead of wavelet decomposition because the wavelet based decomposition of a mesh is difficult to implement and unreliable.

\section{Experimental Results and Discussion}

In this section, the experimental results and quantitative analysis of the performance of 3D ASM, 3D PASM and 3D HASM are presented. The 2D HASM in 2] is extended to 3D HASM based on mesh partitioning in this study to compare with 3D PASM. The 3D HASM uses overlapping tiles and statistics in coarser scales to avoid the inconsistence of shape between tiles.

\subsection{Image Data}

The training sets and images used in this work are from the Internet Brain Segmentation Repository (IBSR) [4], which is an open online database. There are two sets of human brain MR images and their manual segmentations used in this work. The first set is IBSR v1.0. They are 20 normal T1-weighted MR brain $3 \mathrm{D}$ images and their manual segmentations. The second set is IBSR v2.0. They are 18 normal T1-weighted MR brain 3D images and their manual segmentations. The objects to segment are Lateral Ventricles extracted from IBSR v1.0, Lateral Ventricles extracted from IBSR v2.0, Left Thalamus Propers extracted from IBSR v2.0 and Left Hippocampuses extracted from IBSR v2.0. For simplicity, they are called LV1, LV2, LTP and LH in this study, respectively.

These three objects and two sets of images are chosen because of their diversity. Images of IBSR v1.0 are noisy. Images of IBSR v2.0 have higher quality and are free from noise. The contrast of the region around lateral ventricles is 
sharp. On the contrary, the contrast of the regions around thalamus propers and hippocampuses is relatively vague.

\subsection{A Comparative Study of Segmentation Using Various Models}

Standard 3D ASMs, 3D HASMs and 3D PASMs are applied to segment LV1, LV2, LTP and LH from test images. For comparison purpose, the initialization and parameters of the three models are exactly the same. The "leave-one-out" method is used in this experiment. The "segmentation error" is used to measure the accuracy of the segmentations. The error is defined as the distances between the segmentation results and the corresponding manual segmentations. This metric is defined in [5]. In short, it is the shortest distance between two normalized meshes. If a segmentation error is bigger than $1 / 3$ of the initialization error, this segmentation is considered as a "failure" in this study.

Table 1. The average segmentation errors (in voxels)

\begin{tabular}{ccccc}
\hline & LV1 & LTP & LH & LV2 \\
\hline 3D ASM & 2.6364 & 4.6983 & 9.1490 & 1.5470 \\
3D HASM 1.6282 & 2.6014 & 4.4104 & 1.5609 \\
3D PASM & 1.2469 & 1.1717 & 4.2943 & 1.5104 \\
\hline
\end{tabular}

One example of the manual segmentation, initialization and the segmentation results of models are illustrated in Fig. 3. The segmentation by models are overlayed with cross-sections (in gray contours) and the 3D surface of manual segmentations, where the dark surface is the segmentation by the models and the bright one is the manual segmentation. The quantitative results are shown in Fig. 4. Their average is listed in Table 1] More results are available at www.unc.edu/ kurtzhao.

In several cases, the standard 3D ASMs failed to find the desired boundaries, because 19 or 17 samples are not sufficient for ASMs to accurately estimate the distribution of samples. During iterations, the rigidity of ASMs tends to keep model points from moving to approximate appropriate shape details. The accumulation of errors in iterations could lead to segmentation failures. Furthermore, model points have difficulty to find their best match in noisy images. As a result, during iterations, when a few points find their best matches, the shape of the model cannot change accordingly to guide other points, because 3D ASMs are too restrictive.

3D HASMs have partitioned representation, so they do not have this "rigidity" problem. But they still failed to accurately segment in a few cases because they tolerate illegal deformations, i.e. their allowable regions are not plausible. On the contrary, the 3D PASM improved the segmentation accuracy significantly because of the partitioned representation and the model fitting scheme using curve alignment. The improvements by 3D PASMs on LV2 are not as significant as other objects because of the images of LV2 are very clear and the 


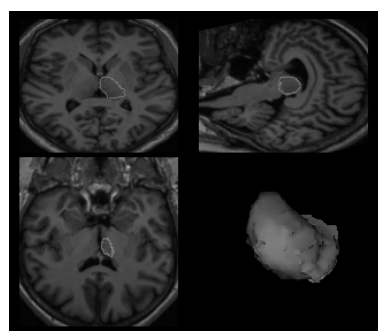

(a) Manual Segmentation

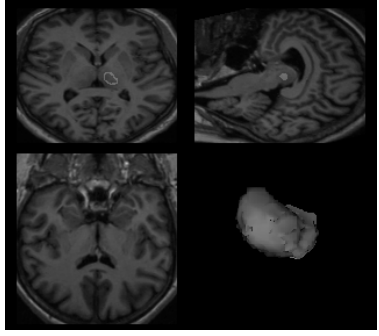

(b) Initialization: 5.5668

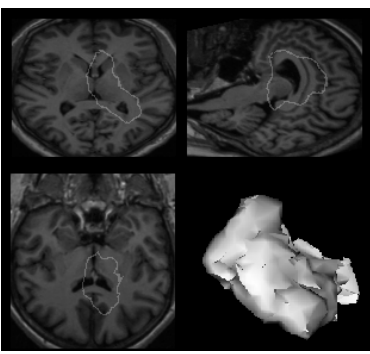

(c) ASM: 10.2965

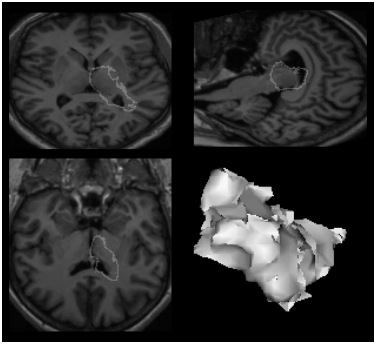

(d) 3D HASM: 5.4502

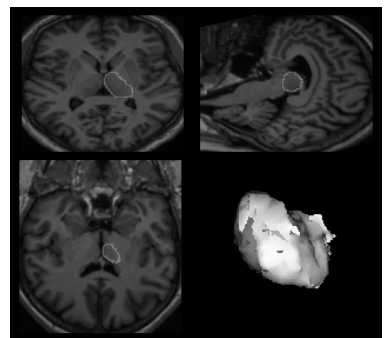

(e) 3D PASM:1.1381

Fig. 3. The comparative study of the accuracy of segmentations for LTP by using different models
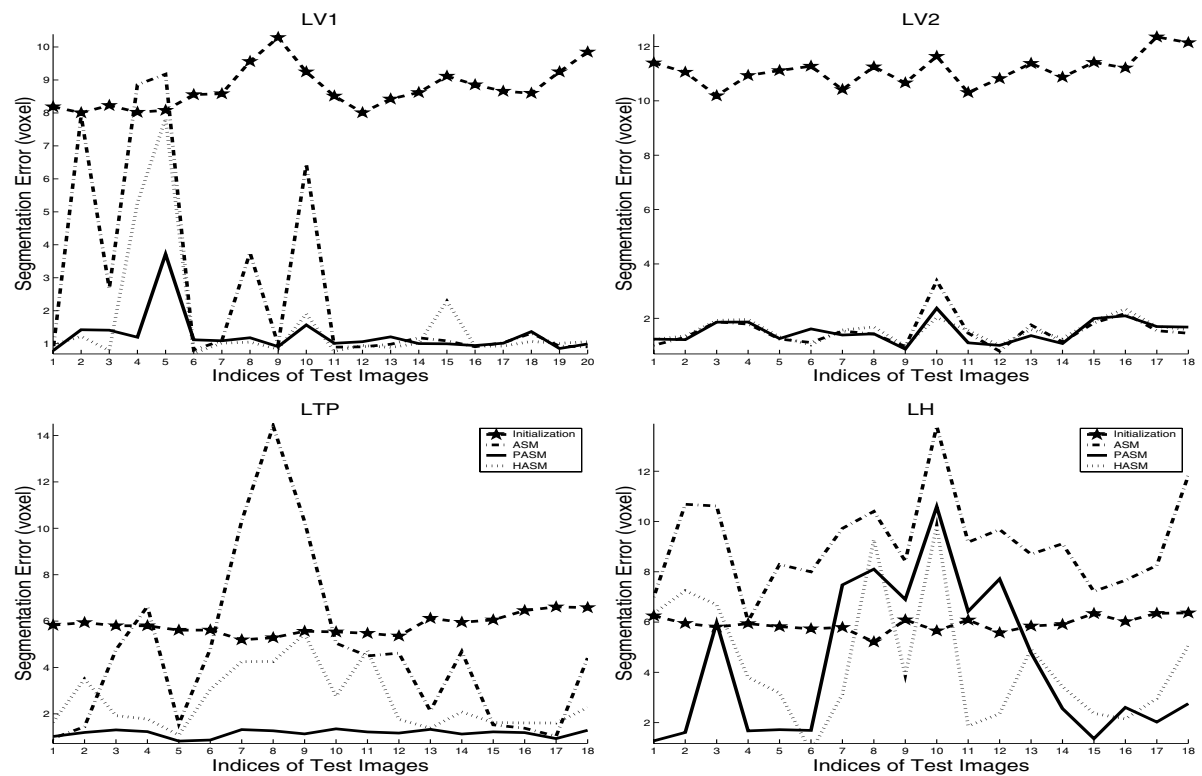

Fig. 4. The quantitative results of segmentations using different models 
lateral ventricles have strong contrasts. However, if the images are noisy or the contrasts are low, 3D PASM brings significant improvements over 3D ASM and 3D HASM.

\section{Conclusion and Discussion}

A 3D Partitioned Active Shape Model is proposed in this work to solve the "short of training samples" problem. The experimental results show that 3D PASM brought significant improvement on the accuracy of segmentation over ASM and HASM. The PASM differs from HASM in how they avoid the inconsistency/discontinuites of shape caused by the mesh partitioning. The HASM uses overlapping tiles, as well statistics in coarser scales. The PASM uses a more systematic approach: considering the statistics of all tiles simultaneously. As the results show, the PASM is more robust to noisy images and low contrasts. Currently, the tiles are constructed so that they have similar numbers of faces. However, it is worth to investigate to take into consideration the shape variation of tiles. There are two extreme conditions: 1) a mesh consisting of $\mathrm{N}$ faces is partitioned into $\mathrm{N}$ tiles; 2) a mesh is partitioned into one tile only. In these two extreme, the PASM is not expected to improve the performance over ASM. However, $K^{\prime}$, the number of tiles is set to avoid these two extremes.

\section{References}

1. Cootes, T.F., Taylor, C.J. In: Statistical Models of Appearance for Computer Vision. Technical Report, University of Manchester (2001)

2. Davatzikos, C., Tao, X., Shen, D.: Hierarchical active shape models, using the wavelet transform. IEEE Transactions on Medical Imaging 22(3) (March 2003) 414-423

3. Ip, H.H.S., Shen, D.: An affine-invariant active contour model (ai-snake) for modelbased segmentation. Image and Vision Computing 16 No.2 (1998) 125-146

4. avaliable at http://neuro www.mgh.harvard.edu/cma/ibsr: (The internet brain segmentation repository (ibsr))

5. Zhao, Z., Teoh, E.K.: A novel framework for automated 3D PDM construction using deformable models. In: Proc. Medical Imaging 2005: Image Processing, Proceedings of SPIE 2005 (SPIE MI 05) (in press). (2005) 